\title{
Therapeutic effect of Rho kinase inhibitor FSD-C10 in a mouse model of Alzheimer's disease
}

\author{
QING-FANG GU ${ }^{1 *}$, JIE-ZHONG YU $^{1 *}$, HAO WU ${ }^{1}$, YAN-HUA LI $^{1}$, CHUN-YUN LIU $^{1}$, \\ LING FENG $^{1}$, GUANG-XIAN ZHANG ${ }^{2}$, BAO-GUO XIAO ${ }^{3}$ and CUN-GEN MA ${ }^{1,4}$
}

\author{
${ }^{1}$ Department of Neurology, Institute of Brain Science, Medical School, Shanxi Datong University, \\ Datong, Shanxi 037009, P.R. China; ${ }^{2}$ Department of Neurology, Thomas Jefferson University, Philadelphia, PA 19107, USA; \\ ${ }^{3}$ Institute of Neurology, Huashan Hospital, Institutes of Brain Science and State Key Laboratory of Medical Neurobiology, \\ Fudan University, Shanghai 200025; ${ }^{4} 2011$ Collaborative Innovation Center/Research Center of Neurobiology, \\ Shanxi University of Traditional Chinese Medicine, Taiyuan, Shanxi 030024, P.R. China
}

Received May 23, 2018; Accepted August 23, 2018

DOI: $10.3892 / \mathrm{etm} .2018 .6701$

\begin{abstract}
Fasudil, a Rho kinase (ROCK) inhibitor, effectively inhibits disease severity in a mouse model of Alzheimer's disease (AD). However, given its significant limitations, including a relatively narrow safety window and poor oral bioavailability, Fasudil is not suitable for long-term use. Thus, screening for ROCK inhibitor(s) that are more efficient, safer, can be used orally and suitable for long-term use in the treatment of neurodegenerative disorders is required. The main purpose of the present study is to explore whether FSD-C10, a novel ROCK inhibitor, has therapeutic potential in amyloid precursor protein/presenilin-1 transgenic (APP/PS1 Tg) mice, and to determine possible mechanisms of its action. The results showed that FSD-C10 effectively improved learning and memory impairment, accompanied by reduced expression of amyloid- $\beta$ 1-42 (A $\left.\beta_{1-42}\right)$, Tau protein phosphorylation (P-tau) and $\beta$-site APP-cleaving enzyme in the hippocampus and cortex area of brain. In addition, FSD-C10 administration boosted the expression of synapse-associated proteins, such as postynaptic density protein 95 , synaptophsin, $\alpha$-amino 3-hydroxy-5-methyl-4-isoxa-zolep-propionate receptor and
\end{abstract}

Correspondence to: Professor Cun-Gen Ma, Department of Neurology, Institute of Brain Science, Medical School, Shanxi Datong University, 1 Xingyun Road, Datong, Shanxi 037009, P.R. China

E-mail: macungen2001@163.com

Professor Bao-Guo Xiao, Institute of Neurology, Huashan Hospital, Institutes of Brain Science and State Key Laboratory of Medical Neurobiology, Fudan University, 138 Yixueyuan Road, Shanghai 200025, P.R. China

E-mail:bgxiao@shmu.edu.cn

*Contributed equally

Key words: Alzheimer's disease, APP/PS1transgenic mice, Rho kinase, FSD-C10 neurotrophic factors, e,g., brain-derived neurotrophic factor and glial cell line-derived neurotrophic factor. Taken together, our results demonstrate that FSD-C10 has therapeutic potential in the AD mouse model, possibly through inhibiting the formation of $A \beta_{1-42}$ and P-tau, and promoting the generation of synapse-associated proteins and neurotrophic factors.

\section{Introduction}

Alzheimer's disease (AD) is a neurodegenerative disorder that occurs mainly in old age; it is characterized by deposits of amyloid- $\beta$ (A $\beta$ ) plaques and neurofibrillary tangles, and neuronal loss (1), and its prevalence is rapidly increasing. It is likely that $\mathrm{AD}$ has multiple etiologies, although its precise cause remains unknown (2). $\mathrm{A} \beta$ and tau proteins constitute a prime neurotoxic component of senile plaques in the brain of $\mathrm{AD}$ patients, thus contributing to learning and memory impairment due to synaptic dysfunction and neuronal degeneration (3). However, to date most therapeutic interventions aimed at modifying a single pathological factor (e.g., cholinergic dysfunction, or A $\beta$ aberrant processing) have failed because they target only limited pathogenic factors of AD (4). Inflammation, mitochondrial dysfunction, and oxidative stress are considered the most prominent concomitant pathological events (5-7), being potential targets of therapeutic intervention. Recently, the ER-associated degradation (ERAD) pathway has also been drawing widespread attention as control of protein-folding intermediaries in $\mathrm{AD}(8,9)$.

Inflammation has been proposed as a main factor in the pathogenesis of $\mathrm{AD}$, including microglial activation, reactive astrocytes and inflammatory molecules (2,10-12). However, it has been noted that microglial activation exhibits both beneficial and detrimental effects depending on the stage of microglia (13). The conversion of microglia from detrimental (M1) to beneficial (M2) phenotype may contribute to an anti-inflammatory microenvironment in the brain (14). Similar to microglia, astrocytes also contribute to neuroinflammation in $\mathrm{AD}$ by releasing inflammatory cytokines and other toxic molecules (15). The ubiquitin-proteasome system and autophagy mechanisms are impaired due to the toxic effects of 
$\mathrm{A} \beta$ and oxidative stress damage, leading to the accumulation of oxidized/unfolded proteins that may contribute to neuronal loss (16). In fact, non-steroidal anti-inflammatory drugs $\left(\mathrm{NSAID}_{\mathrm{S}}\right)$ initially garnered enthusiasm from pre-clinical and epidemiologic studies as agents for reducing the risk of AD (17), but anti-inflammatory treatment failed to produce beneficial effects in patients with severe cognitive impairment and dementia (18).

It has been reported that Rho activity, which is thought to contribute to AD pathogenesis (19), was elevated in the brain of AD model mice (20). Pharmacologic inhibition of Rho kinase (ROCK) induced protein degradation by autophagy in mammalian cells (21), and suppressed $A \beta$ production in an AD mouse model (22), highlighting ROCK as a therapeutic target to combat $\mathrm{A} \beta$ production in AD. Fasudil, a selective ROCK inhibitor, increased dendrite branching and stabilized dendrite arbors in CA1 pyramidal neurons of APP/PS1 mice (23) by preventing neurodegeneration and stimulating neuroregeneration in various neurological disorders (19). Our previous study also confirmed that Fasudil treatment ameliorated memory deficits in APP/PS1 transgenic mice, accompanied by a decrease in $A \beta$ deposits, $p$-Tau and BACE levels, an increase in PSD-95, and inhibition of the TLRS-NF- $\mathrm{B}-\mathrm{MyD} 88$ inflammatory cytokine axis (24).

Although previous studies have demonstrated certain beneficial effects of Fasudil intervention in the AD model (24), several lines of evidence suggest that there are some limitations in the clinical use of Fasudil, including its suitability only for short-course treatment, low oral bioavailability, a narrow safety window and blood pressure fluctuation. Thus, novel ROCK inhibitor(s) that are more efficient, safer, oral and suitable for long-term use for the treatment of neurodegenerative disorders are required. In the present study, we explored the therapeutic effect and systemic response of a novel ROCK inhibitor, FSD-C10, and possible mechanisms of its action in the treatment of a mouse model of AD.

\section{Materials and methods}

Animals and treatment. Experiments were performed on male APP/PS1 double transgenic mice (APPswe/PS1 $1_{\mathrm{dE} 9}$, 8-month-old), purchased from Shanghai Research Center. Age-matched wild-type (WT) mice were used as controls. All animals were housed in a room maintained at $25^{\circ} \mathrm{C}$ with a 12-h light/dark cycle. The mice were given free access to food and water except during the behavioral test. The experiment was carried out in compliance with the Guidelines for Animal Care and Use of China, and approved by the Animal Ethics Committee of Shanxi Datong University, Datong, China (Ethical Approval no. 1601). Every effort was made to minimize suffering of the animals.

The experimental design was carried out in two stages: Validation of the AD model and intervention of the AD model. For validation of the AD model, mice were divided into two groups: Age-matched wild-type $(n=8)$ and APP/PS1 transgenic mice $(n=8)$. Mice were sacrificed at 8 months of age. Behavioral and pathological changes were measured before and after execution. For the intervention of FSD-C10, mice were divided into two groups: Normal saline (NS)-control mice $(n=8)$ and FSD-C10-treated mice $(n=8)$. Mice were treated with FSD-C10 (25 mg/kg/day every other day) or NS $(0.9 \% \mathrm{NaCl})$ for 2 months by intraperitoneal (i.p.) injection. The concentration of FSD-C10 used in this study was adopted based on our preliminary experiments.

Mouse spatial learning and memory test. Spatial learning and memory of mice were assessed using the Morris water maze (MWM) test. The MWM is a $90 \mathrm{~cm}$ high, $50 \mathrm{~cm}$ diameter circular pool, containing a submerged escape platform $(5.0 \times 5.0 \mathrm{~cm}), 2.0 \mathrm{~cm}$ below the water surface. The pool was filled with water containing an edible white pigment that made the water opaque, and the water temperature was maintained at $23-25^{\circ} \mathrm{C}$. In each trial, the time required to escape onto the hidden platform was recorded as escape latency. Mice were allowed a maximum of $60 \mathrm{sec}$ to reach the platform, and if they failed to do so, they were guided to the platform. After training was completed, cognitive function was measured for 5 days, after which the platform was removed for spatial exploration in order to determine the memory capacity of the mouse as to the platform space position. All behavioral parameters of mice were tracked, recorded and analyzed using SMART 3.0 (Panlab, Barcelona, Spain).

Histopathology and immunohistochemistry. At the end of the final behavioral test, mice were anesthetized and transcardially perfused with NS and 4\% paraformaldehyde in phosphate-buffered saline (PBS). Brains were sliced $(10 \mu \mathrm{m})$ and analyzed by immunohistochemistry. Briefly, slides were blocked with $1 \%$ BSA (Sigma) for $1 \mathrm{~h}$, and permeated with $0.3 \%$ Triton X-100 in $1 \% \mathrm{BSA} / \mathrm{PBS}$ for $30 \mathrm{~min}$ at RT. Sections were incubated with anti-A $\beta_{1-42}(1: 1,000 ;$ EMD Millipore, Billerica, MA, USA), anti-p-Tau (1:1,000; Cell Signaling Technology, Inc., Danvers, MA, USA), anti-BACE (1:1,00; Cell Signaling Technology, Inc.), anti-synaptophin (1:1,000), anti-AMPAR-1 (1:1,000), anti-AMPAR-2 (1:1,000; all from Abcam, Cambridge, MA, USA), anti-BDNF (1:1,000; Promega Corporation, Madison, WI, USA), and anti-GDNF (1:1,000; Promega Corporation) at $4^{\circ} \mathrm{C}$ overnight. After washing, the slices were incubated with secondary antibodies at RT for $2 \mathrm{~h}$. The nucleus was stained by Hoechst 33342 (1 $\mu \mathrm{g} / \mathrm{ml}$, Sigma-Aldrich; Merck KGaA, Darmstadt, Germany). Control sections were run following identical protocols, but the primary antibodies were omitted. Results were visualized under fluorescence microscopy by IMAGE-PRO PLUS software (Media Cybernetics, Inc., Rockville, MD, USA). Quantification analysis of positive cells was performed on three sections per animal, and four mice per group were analyzed in a blinded fashion.

Western blot analysis. Proteins of mouse brain were extracted as previously described (17), Protein concentrations were determined by a bicinchoninic acid (BCA) kit (Beyotime Institute of Biotechnology, Haimen, China). Protein samples were separated by SDS-PAGE and transferred onto PVDF membranes. After nonspecific binding was blocked with 5\% nonfat dry milk, membranes were incubated at $4^{\circ} \mathrm{C}$ overnight with primary antibodies $\left(\mathrm{A} \beta_{1-42}, \mathrm{p}-\mathrm{Tau}, \mathrm{BACE}, \mathrm{PSD}-95\right.$, AMPAR-1, AMPAR-2, BDNF, GDNF and $\beta$-actin). The next day, the membranes were washed three to four times with $0.1 \%$ Tween-20/TBST ( $\mathrm{pH}$ 7.6) and incubated with horseradish peroxidase-conjugated anti-rabbit $\mathrm{IgG}$ or anti-mouse 

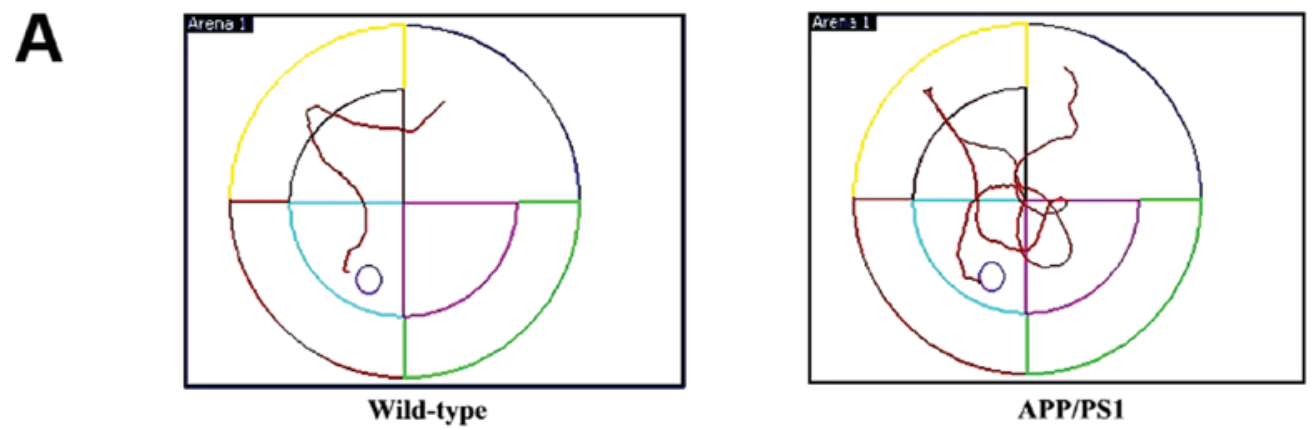

B
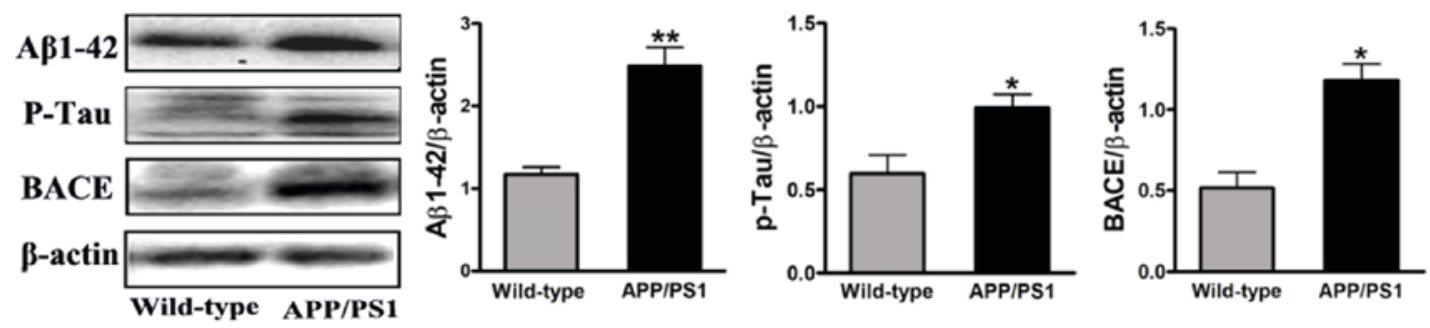

Figure 1. AD transgenic mouse model. At 8 months of age, learning and memory ability was measured by the Morris Water Maze. (A) Compared with the WT group, APP/PS1 transgenic mice exhibited obvious space learning and memory impairment. (B) At the same time, expression of A $\beta_{1-42}$, p-Tau and BACE protein was increased as measured by western blot. The results suggest that APP/PS1 mice showed typical behavior dysfunction and and typical pathologic abnormality of AD. Results are expressed as the fold change relative to $\beta$-actin as the loading control. Quantitative results are shown as mean \pm SEM of 4 mice each group. ${ }^{*} \mathrm{P}<0.05$ and ${ }^{* *} \mathrm{P}<0.01$ vs. Wild-type. AD, Alzheimer's disease.

$\operatorname{IgG}$ secondary antibodies for $2 \mathrm{~h}$ at $37^{\circ} \mathrm{C}$. To compare protein loading, antibodies directed against GAPDH or $\beta$-actin were used and relative optical density was measured with Image Lab Software (Bio-Rad Laboratories, Inc., Hercules, CA, USA).

Statistical analysis. Graph Pad Prism 5 (Cabit Information Technology Co., Ltd., Shanghai, China) was used for statistical analysis. Results are presented as mean \pm SEM. An unpaired, two-tailed Student's t-test was used for comparisons of means between two groups. In all tests, $\mathrm{P}<0.05$ was considered to indicate a statistically significant difference.

\section{Results}

Behavioral and pathological changes in APP/PS1 mice at 8 months of age. To observe the effect of FSD-C10 in APP/PS1 transgenic mice, we first tested the behavioral and pathological changes at the initiation time of drug intervention as a baseline. Memory and learning ability was measured by MWM and the expression of $\mathrm{A} \beta_{1-42}, \mathrm{p}$-Tau and BACE protein was determined by western blot. As shown in Fig. 1, APP/PS1 transgenic mice exhibited obvious space learning and memory impairment at 8 months of age, as compared with the WT group. Simultaneously, the levels of $A \beta_{1-42}$, p-Tau and BACE expression were more elevated in APP/PS1 transgenic mice than in the WT group $(\mathrm{P}<0.01, \mathrm{P}<0.05$ and $\mathrm{P}<0.05$, respectively; Fig. 1B). Together, APP/PS1 mice showed the typical behavior dysfunction and typical pathologic abnormality of $\mathrm{AD}$, thus confirming the AD model in these transgenic mice.

FSD-C10 improves learning and memory abilities of the APP/PS1 Tg mice. As shown in Fig. 1, APP/PS1 transgenic mice had obvious space learning and memory impairment at
8 months of age compared with the WT group. To observe whether FSD-C10 can ameliorate the deficit in learning and memory ability, MWM measurement was used in APP/PS1 mice after 2 months of treatment (Fig. 2A and B). As shown in Fig. 2C, FSD-C10 intervention significantly reduced the time and distance for Latency to Target $(\mathrm{P}<0.01)$, Latency 1st Entrance to SW $(\mathrm{P}<0.05)$, and mean distances to Target $(\mathrm{P}<0.05)$ of these AD mice.

When the platform was moved, a consolidation of spatial memory was detected. The results showed that the movement of FSD-C10-treated mice mainly located on the position of the target platform quadrant, while NS-controlled mice mainly moved around the location of the target platform quadrant (Fig. 2A and B). The intervention of FSD-C10 increased Time in SW $(\mathrm{P}<0.01$; Fig. 2C) and Distance in SW ( $<<0.05$; Fig. 2C) as compared with the NS control group. However, there were no differences in global activity between two groups ( $\mathrm{P}>0.05$; Fig. 2C).

FSD-C10 attenuates A $\beta$ burden, Tau phosphorylation and $B A C E$ expression. We evaluated the pathological changes in APP/PS1 transgenic mice, including A $\beta$ plaques, Tau phosphorylation and BACE expression by immunohistochemistry and western blot. As shown in Fig. 3A, the numbers of $\mathrm{A} \beta_{1-42}$-positive cells in the hippocampus and cortex of brain were lower in FSD-C10-treated mice than in NS-treated control mice. Similarly, FSD-C10 intervention suppressed $A \beta_{1-42}$ protein level in brain when compared with the NS-treated control group $(\mathrm{P}<0.01$; Fig. 3B).

We further explored the levels of p-tau and BACE proteins in the hippocampus and cortex with immunohistochemistry and western blot. The results showed that the numbers of p-Tau and BACE positive cells were significantly lower in FSD-C10-treated group than in the NS-treated control group 
A

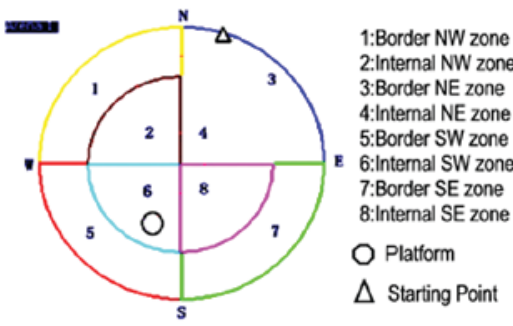

8 zone morris water maze

C
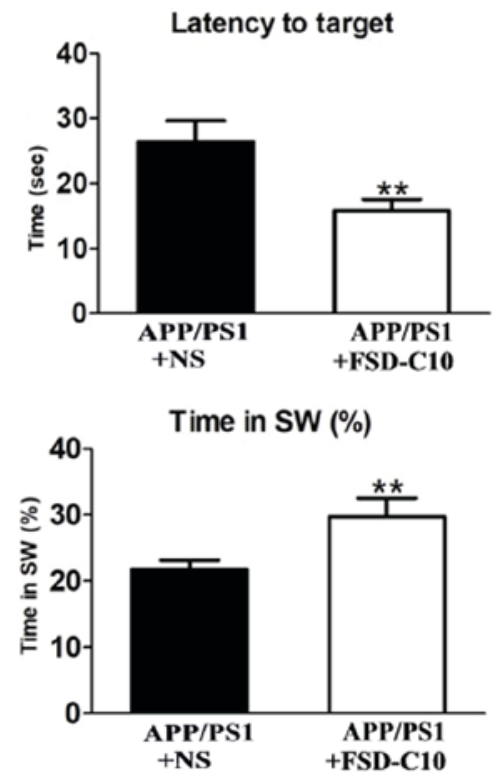

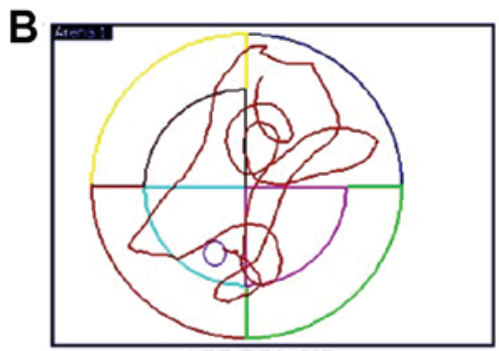

APP/PS1+NS
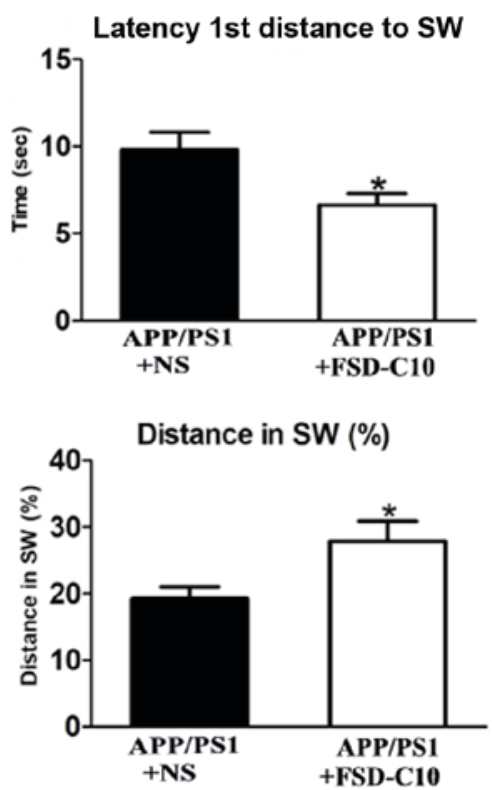

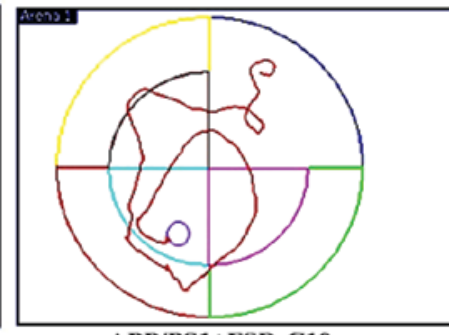

APP/PS1+FSD-C10
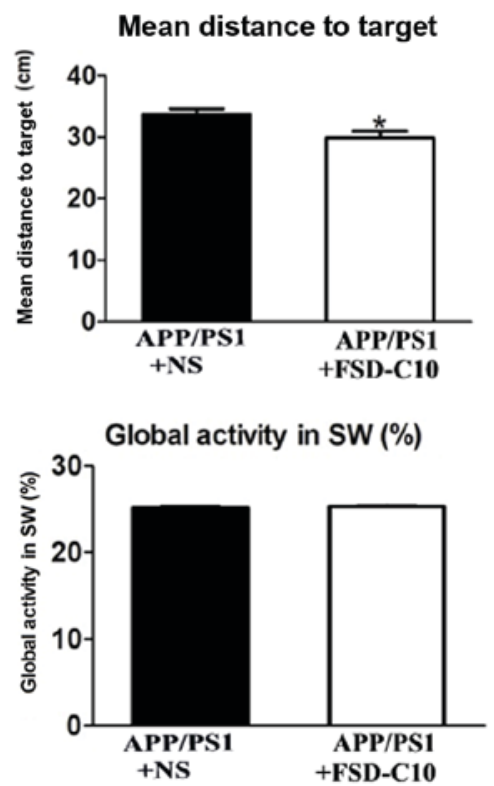

Figure 2. FSD-C10 improves behavioral deficits of APP/PS1 Tg mice. Cognitive ability was analyzed in a Morris water maze test. The Morris water maze test began two months after NS/FSD-C10 administration. (A) 8-zone Morris water maze schematic diagram, (B) typical diagram of two groups, and (C) Latency to Target, Latency 1st Entrance to SW, Mean Distances to Target, Time in SW (\%), Distance in SW and Global Activity (\%). Results are shown as mean \pm SEM of 8 mice each group. ${ }^{*} \mathrm{P}<0.05$ and ${ }^{* *} \mathrm{P}<0.01$ vs. APP/PS1+NS.

$(\mathrm{P}<0.05$ and $\mathrm{P}<0.01$ for $\mathrm{p}-\mathrm{Tau} ; \mathrm{P}<0.05$ and $\mathrm{P}<0.05$ for $\mathrm{BACE}$; Fig. 4A). Similarly, FSD-C10 treatment inhibited the expression of $\mathrm{p}$-Tau and BACE protein in brain when compared with the NS-treated control mice $(\mathrm{P}<0.05$; Fig. 4B). These results suggest that FSD-C10 treatment effectively alleviated pathological changes in $\mathrm{AD}$ mice.

FSD-C10 induces the expression of synapse-associated proteins. It has become clear that cognitive dysfunction more strongly correlates with synapse loss in AD than with counts of plaques, tangles, and neuronal loss $(25,26)$. Our results showed that FSD-C10 treatment up-regulated synaptophsin expression in the hippocampus $(\mathrm{P}<0.01$; Fig. 5A) as well as AMPAR-1 and AMPAR-2 expression in the cortex $(\mathrm{P}<0.01$ and $\mathrm{P}<0.05$, respectively; Fig. 5A) as compared with NS-treated control mice. Data from western blot also confirmed that FSD-C10 treatment up-regulated the expression of AMPAR-1 and AMPAR-2 protein in brain (both $\mathrm{P}<0.01$; Fig. $5 \mathrm{~B}$ ).

PSD-95 is a synaptic protein that regulates synaptic distribution, synaptic stability and certain types of memory (27). As shown in Fig. 5B, FSD-C10 treatment elevated the level of PSD-95 expression in brain as compared with the NS-treated control group $(\mathrm{P}<0.05$; Fig. $5 \mathrm{~B})$. These results indicate that the improvement in learning and memory impairment mediated by FSD-C10 may be related to the up-regulation of these synapse-associated proteins in both hippocampus and cortex.

FSD-C10 increases the expression of neurotrophic factors. Neurotrophic factors are well known to be important for the survival, differentiation, growth and regeneration of neurons (28), as well as for synaptic transmission and plasticity (29). In this research, we explored the role of FSD-C10 in production of neurotrophic factors. Immunohistochemical analysis revealed that FSD-C10 intervention increased the numbers of both BDNF and GDNF positive cells in the hippocampus and cortex of brain compared with the NS-treated control group $(\mathrm{P}<0.01$ and $\mathrm{P}<0.05$ respectively; Fig. 6A). Expression of BDNF and GDNF protein was also higher in FSD-C10-treated mice than in the NS-treated control group measured by western blot (both $\mathrm{P}<0.05$; Fig. $6 \mathrm{~B}$ ). FSD-C10 thus has a potentially neuroprotective effect through the production of neurotrophic factors.

\section{Discussion}

$\mathrm{AD}$ is an age-related and chronic neurodegenerative disorder presenting as progressive cognitive decline. Although the exact pathogenesis is not yet clear, multiple etiologic pathways have 
A

A $\quad$ Cortex
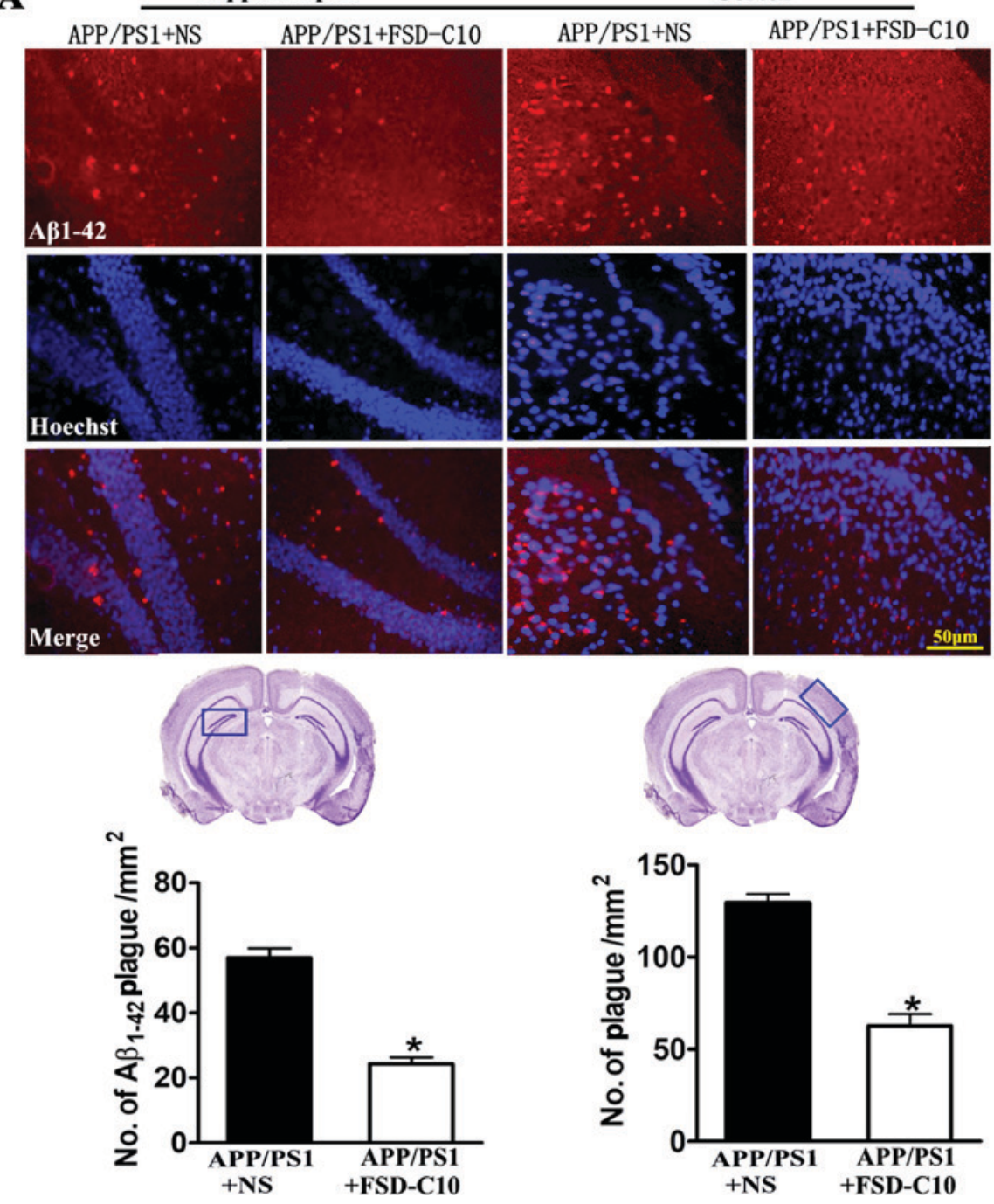

B

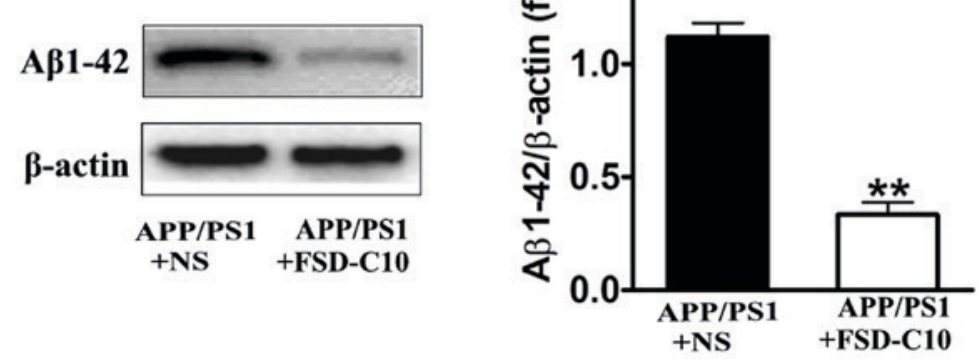

Figure 3. FSD-C10 attenuates $\mathrm{A} \beta_{1-42}$ burden in hippocampus and cortex in APP/PS1 Tg mice. A $\beta_{1-42}$ was measured by immunohistochemistry and western blot on two months after FSD-C10 administration. (A) $\mathrm{A} \beta_{1-42}$ expression was measured in hippocampus and cortex by immunohistochemistry and (B) in brain tissue by western blot. Quantitative results are shown as mean \pm SEM of 4 mice each group, and one representative of three independent experiments. ${ }^{*} \mathrm{P}<0.05$ and $^{* *} \mathrm{P}<0.01$ vs. APP/PS1+NS.

been considered (2). The major histopathological hallmarks of $\mathrm{AD}$ include $\mathrm{A} \beta$ plaques and neurofibrillary tangles (NFTs) formed by hyperphosphorylated Tau protein (30), and the neurite atrophy and synaptic loss induced by $\mathrm{A} \beta$ are considered to be the major cause of gradual cognitive detetrioration in AD (31). Neurotoxicity of $A \beta$ becomes apparent via induction of oxidative stress, neuronal excitotoxicity, neuroinflammation and apoptosis $(32,33)$. One of the most promising strategies for treatment of $\mathrm{AD}$ is to directly target $\mathrm{A} \beta$ by decreasing the production and clearing aggregation of $\mathrm{A} \beta$ (34). In addition to $\mathrm{A} \beta$ accumulation, tau hyperphosphorylation is also an important pathological hallmark of AD (35). Abnormal phosphorylation of tau protein promotes the loss of microtubule stabilizing ability and may contribute to neurite degeneration as well as NFT formation (36). Current drugs for AD treatment, such as acetylcholinesterase inhibitor and NMDA antagonist, show limited benefits in most AD patients (37). There is thus an urgent need for novel therapeutic strategies that can halt the 
A P-Tau $\quad$ BACE
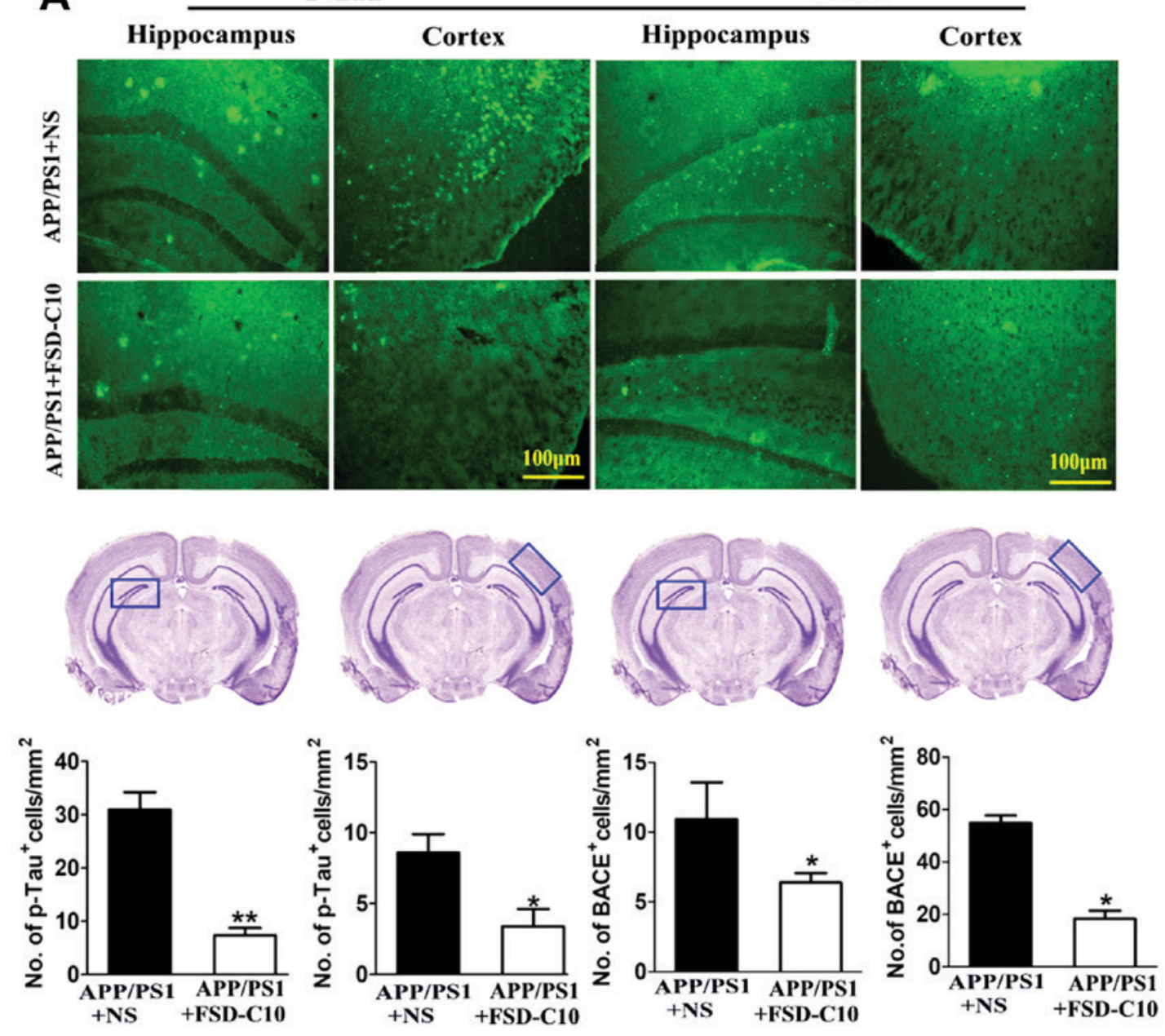

B

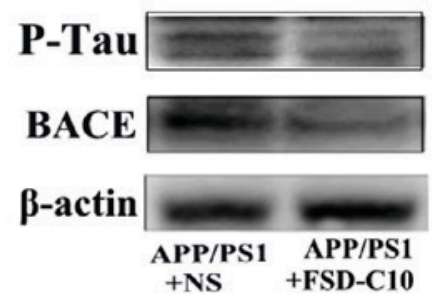

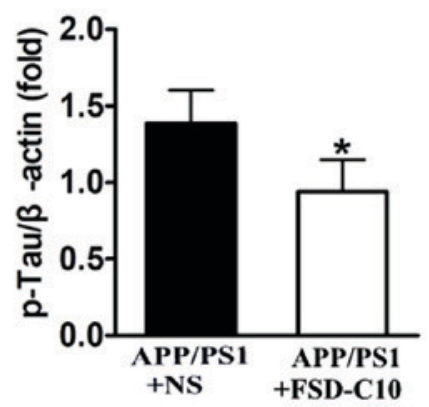

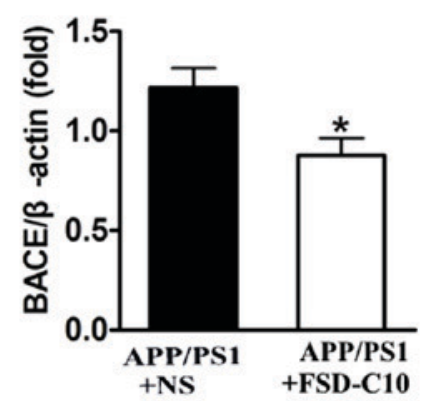

Figure 4. FSD-C10 reduces Tau phosphorylation and BACE expression in hippocampus and cortex in APP/PS1 Tg mice. p-Tau and BACE were measured by immunohistochemistry and western blot two months after FSD-C10 administration. (A) The numbers of p-Tau and BACE positive cells in hippocampus and cortex were measured by immunohistochemistry and (B) their protein expression was measured in brain by western blot. Quantitative results are shown as mean \pm SEM of 4 mice each group, and one representative of three independent experiments. ${ }^{*} \mathrm{P}<0.05$ and ${ }^{* * *} \mathrm{P}<0.01$ vs. APP/PS1+NS.

disease process and are suitable for long-term clinical management. In our previous studies, a comparative study of Fasudil and FSD-C10 has been reported, including cell viability, cell death, neurite outgrowth and dendritic formation, vasodilation insensitivity and animal mortality (38). In this study, we demonstrate that treatment with FSD-C10, as a new ROCK inhibitor, was able to reverse cognitive impairment, possibly through decreasing $\mathrm{A} \beta$ accumulation and Tau phosphorylation in the cortex and hippocampus of APP/PS1 transgenic mice.

A series of studies have demonstrated that ROCK was elevated in the brain of AD patients compared to controls $(39,40)$, and inhibition of ROCK activity decreased A $\beta$ levels by enhancing APP protein degradation (41). Abnormal activation of ROCK has also been found in AD experimental models, and may be involved in the occurrence and development of diseases (41). ROCK activation increased A $\beta$ production, CRMP- 2 phosphorylation and hindered tubulin assembly, leading to the inhibition of synapses $(20,42)$. Inhibition of ROCK reduced the production of $A \beta$ (41) and increased the synaptic density and length of hippocampal pyramidal neurons (43). Fasudil has been proved to protect against nerve degeneration induced by $\mathrm{A} \beta$, and to improve spatial memory and learning ability in AD 

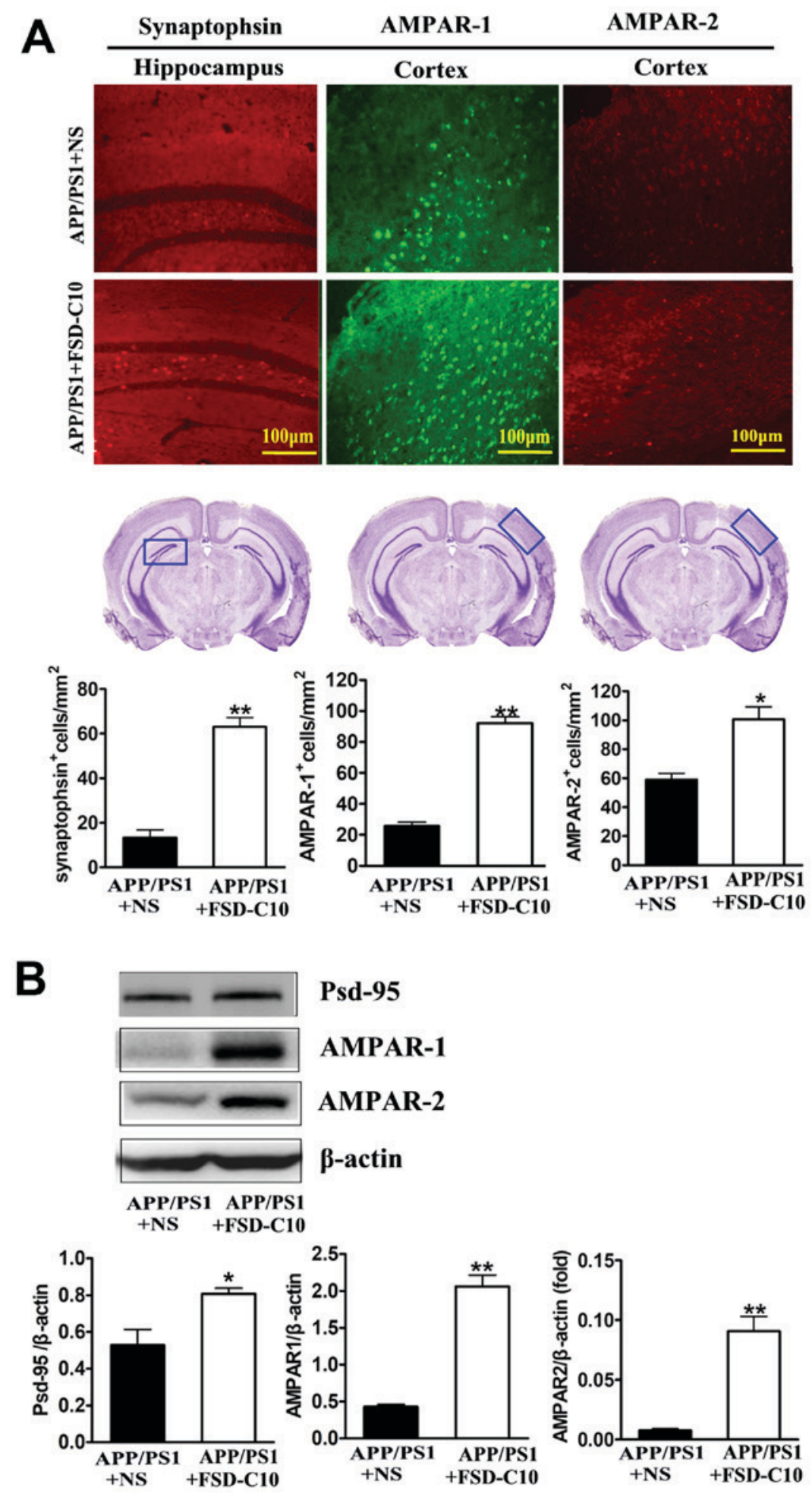

Psd-95

AMPAR-1

AMPAR-2

\section{$\beta$-actin}
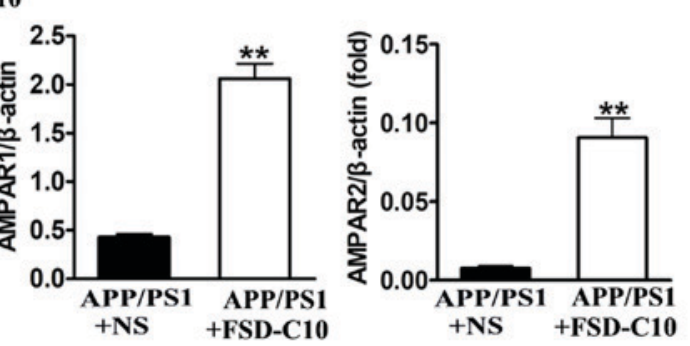

Figure 5. FSD-C10 induces the expression of synaptophsin, AMPAR-1, AMPAR-2 and PSD-95 protein in hippocampus or cortex in APP/PS1 Tg mice. Synaptophsin, AMPAR-1, AMPAR-2 and PSD-95 were measured by immunohistochemistry and western blot two months after FSD-C10 administration. (A) The numbers of synaptophsin, AMPAR-1 and AMPAR-2 positive cells in hippocampus or cortex were measured by immunohistochemistry; (B) their protein in brain was measured by western blot. Quantitative results are shown as mean \pm SEM of 4 mice each group, and one representative of three independent experiments. ${ }^{*} \mathrm{P}<0.05$ and ${ }^{* *} \mathrm{P}<0.01$ vs. APP/PS1+NS.

rats (44). Our present study provides further evidence that inhibiting ROCK activity can improve cognitive deficits in APP/PS1 transgenic mice.

In clinical practice, Fasudil has several limitations, including a relatively narrow safety window, poor oral bioavailability, and unsuitability for long-term use. Researchers are looking for novel ROCK inhibitors that are more efficient and safer, can be taken orally and over a long period of time for the treatment of neurodegenerative disorders. We have in previous studies found that FSD-C10, as a novel ROCK inhibitor, has the same therapeutic effect, but is safer than Fasudil (38). In EAE, FSD-C10 ameliorated the clinical severity of disease, 

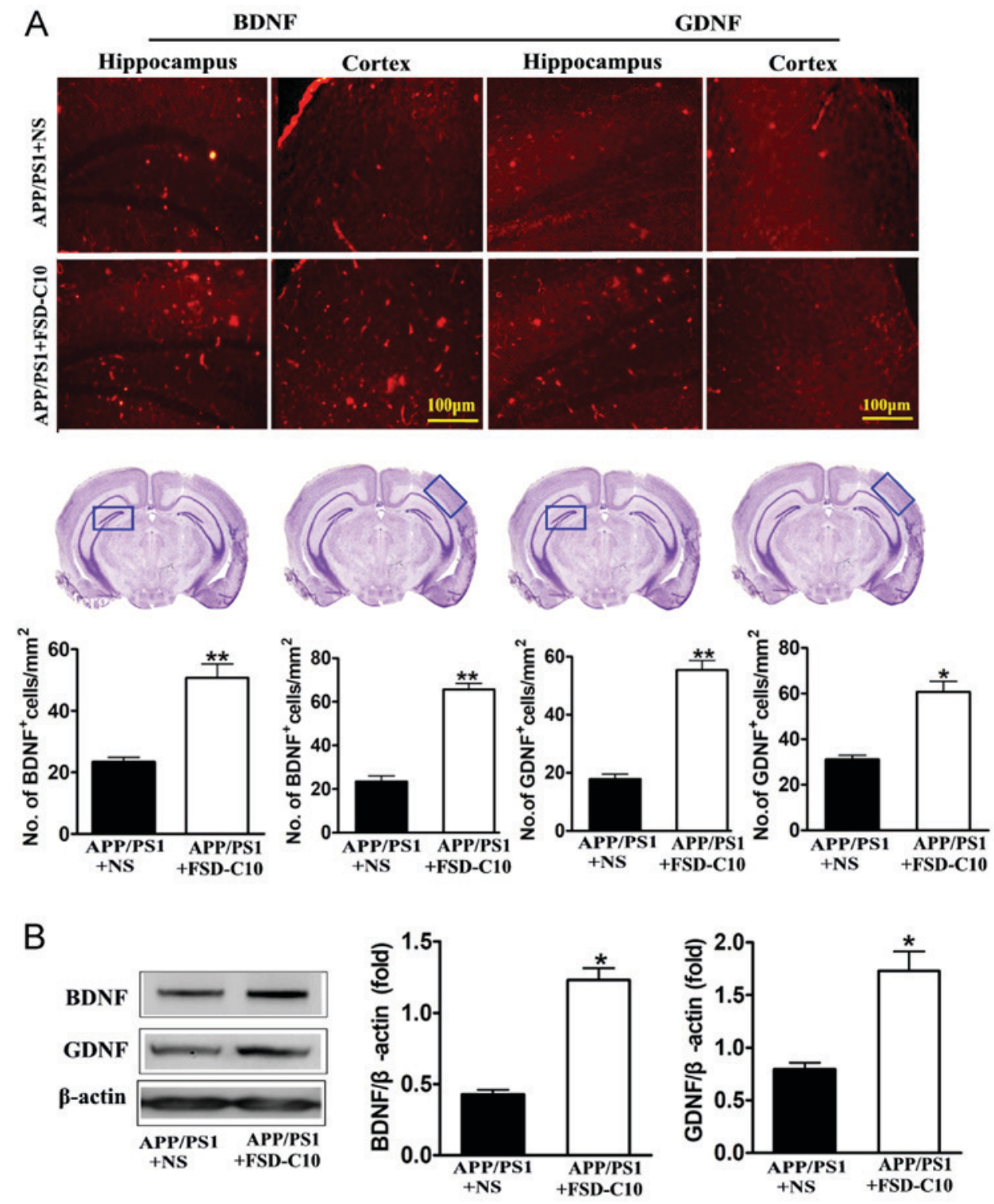

Figure 6. FSD-C10 promotes the expression of neurotrophic factors in hippocampus or cortex in APP/PS1 Tg mice. The expression of BDNF and GDNF protein was measured by immunohistochemistry and western blot two months after FSD-C10 administration. (A) The numbers of BDNF and GDNF positive cells in hippocampus and cortex by immunohistochemistry; and (B) their protein expression in brain by western blot. Quantitative results are shown as mean \pm SEM of 4 mice each group, and one representative of three independent experiments. ${ }^{*} \mathrm{P}<0.05$ and ${ }^{* * *} \mathrm{P}<0.01 \mathrm{vs}$. APP/PS1+NS.

accompanied by improvement in demyelination and the inhibition of inflammatory cells in the CNS of EAE mice, clearly showing a therapeutic effect (45).

Glutamate, the major excitatory neurotransmitter in the $\mathrm{CNS}$, is involved in synaptic transmission, neuronal growth and differentiation, synaptic plasticity and learning and memory (46). When a neuron is depolarized, glutamate is released into the synaptic cleft where it binds glutamate receptors (47). Glutamate receptors, such as NMDA receptor and AMPA receptor, are involved in rapid excitatory synaptic transmission and the release of neurotransmitters, which is closely related with learning and memory (48). In the postsynaptic membrane, an AMPAR insert can induce and promote learning and memory behavior. A $\beta$-induced decline in AMPAR number and synaptic function through endocytosis is a plausible mechanism for the cognitive impairment that occurs in the very early stages of AD (49). Further study found that lack of AMPAR can cause dendritic spine reduction and loss of NMPAR (50), both of which are related to cognitive impairment. In our study, increased expression of AMPAR, synaptophsin and PSD-95 protein after FSD-C10 administration could be an important mechanism for the improvement in cognitive function in transgenic APP/PS1 mice. PSD-95 organizes synaptic proteins to mediate the functional and structural plasticity of the excitatory synapse and to maintain synaptic homeostasis (51). The stabilization of a new synaptic protrusion is associated with activity-driven PSD proteinaceous network formation. In this proteinaceous network, PSD-95 is believed to play a role in synapse maturation, given that it is particularly vulnerable to the toxic effects of $A \beta$ (28). Synaptophysin is the major integral membrane protein of presynaptic vesicles required for vesicle formation and exocytosis (52). Taken together, FSD-C10 may maintain the normal function of the synapse, 
possibly through promoting the expression of these synaptic related proteins.

Neurotrophic factors play an essential role in the survival of neurons affected by degenerative processes $(53,54)$. Increased levels of BDNF are associated with improved learning and memory, and a reduction in BDNF leads to age-related memory deficits (55). The potential of GDNF against age-related cognitive deterioration has not been fully explored. It was reported that serum GDNF levels were significantly reduced in $\mathrm{AD}$ patients $(56,57)$, and expression of GDNF transgene in astrocytes improved cognitive deficits in aged rats (58). Thus, increased BDNF, GDNF, AMPAR, PSD-95, or synaptophysin levels may reflect increased synaptic density, activity, and vesicles, revealing improved functioning of synapses. Inducing expression of these molecules could therefore be a mechanism underlying improved learning and memory abilities of the APP/PS1 transgenic mice after FSD-C10 treatment. The limitations of this study may include that further mechanisms underlying the inhibitory effect on $\mathrm{A} \beta$ after blocking ROCK activity in AD remain unknown, and the oral effect of this reagent in $\mathrm{AD}$ mice needs be tested. These questions will be further explored in the near future.

\section{Acknowledgements}

The authors would like to thank Ms. Katherine Regan (Department of Neurology, Thomas Jefferson University) for editorial assistance.

\section{Funding}

The present study was supported by grants from The National Natural Science Foundation of China (grant nos. 81272163, 81471412 and 81371414), Shanxi University of Traditional Chinese Medicine (grant no. 2011PY-1), research projects supported by Datong Municipal Science and Technology Bureau (grant no. 2017136) and Shanxi Datong University (grant no. 2016K10), and an open project of The State Key Laboratory of Molecular Developmental Biology of China (grant no. 2018-MDB-KF-07 to YQY).

\section{Availability of data and materials}

The analysed data sets generated during the present study are available from the corresponding author on reasonable request.

\section{Authors' contributions}

QFG and JZY designed the study, performed the mouse behavioral tests, participated in the statistical analysis and revised the manuscripts. CGM conceived the study, participated in its design and coordination, and helped to draft the manuscript. BGX designed and performed all analyses, and drafted and revised the manuscript. GXZ participated in the study design and revised the manuscript. YHL performed the immunoassays. CYL performed the western blot analysis. HW and LF performed the statistical analysis and the immunohistochemistry. All authors read and approved the final manuscript.

\section{Ethics approval and consent to participate}

The experiment was carried out in compliance with the Guidelines for Animal Care and Use of China, and approved by the Animal Ethics Committee of Shanxi Datong University, Datong, China (Ethical Approval no. 1601).

\section{Patient consent for publication}

Not applicable.

\section{Competing interests}

The authors declare that they have no competing interests.

\section{References}

1. Goedert M and Spillantini MG: A century of Alzheimer's disease. Science 314: 777-781, 2006.

2. Bou Khalil R, Khoury E and Koussa S: Linking multiple pathogenic pathways in Alzheimer's disease. World J Psychiatry 6: 208-214, 2016.

3. Ferrer I: Defining Alzheimer as a common age-related neurodegenerative process not inevitably leading to dementia. Prog Neurobiol 97: 38-51, 2012.

4. Scheltens P, Blennow K, Breteler MM, de Strooper B, Frisoni GB, Salloway S and Van der Flier WM: Alzheimer'sdisease. Lancet 388: 505-517, 2016.

5. Jiang T, Sun Q and Chen S: Oxidative stress: A major pathogenesis and potential therapeutic target of antioxidative agents in Parkinson's diseaseand Alzheimer's disease. Prog Neurobiol 147: 1-19, 2016.

6. Onyango IG, Dennis J and Khan SM: Mitochondrial dysfunction in alzheimer's disease and the rationale for bioenergetics based therapies. Aging Dis 7: 201-214, 2016.

7. Ransohoff RM: How neuroinflammation contributes to neurodegeneration. Science 353: 777-783, 2016.

8. Cai Y, Arikkath J, Yang L, Guo ML, Periyasamy P and Buch S: Interplay of endoplasmic reticulum stress and autophagy in neurodegenerative disorders. Autophagy 12: 225-244, 2016.

9. Duran-Aniotz C, Cornejo VH and Hetz C: Targeting endoplasmic reticulum acetylation to restore proteostasis in Alzheimer's disease. Brain 139: 650-652, 2016.

10. Hoeijimakers L, Heinen Y, van Dam AM, Lucassen PJ and Korasi A: Microglial priming and Alzheimer's disease: A possible Role for (Early) immune challenges and epigenetics? Front Hum Neurosci 10: 398, 2016

11. Olsen I and Singhrao SK: Inflammation involvement in alzheimer's disease. J Alzheimer's Dis 54: 45-53, 2016

12. Torika N, Asraf K, Roasso E, Danon A and Fleisher-Berkovich S: Angiotensin converting enzyme inhibitors ameliorate braininflammation associated with microglial activation: Possible implication for Alzheimer's disease. J Neueoimmune Pharmacol 11: 774-785, 2016.

13. Wallker DG and Lue LF: Immune phenotypes of microglial in humane neurodegenerativedisease: Challenges to detecting microglial polarization in human brains. Alzheimers Res Ther 7: 56, 2015.

14. Heneka MT, Kummer MP, Stutz A, Delekate A, Schwartz S, Vieira-Saecker A, Gripe A, Axt D, Remus A, Remus A, et al: NLRP3 is actived in Alzheimer's disease and contributes to pathology in APP/PS1mice. Nature 493: 674-678, 2013.

15. Glass CK, Saijo K, Winner B, Marchetto MC and Gage FH: Mechanisms underlying inflammation in neurodegeneration. Cell 140: 918-934, 2010.

16. Trammtola A, Di Domenico F, Barone E, perluigi M and Butterfield DA: It is all about (U)biquitin: role of altered ubiquitin-proteasome system and UCHLI in Alzheimer's Disease. Oxid Med Cell Longev 2756068, 2016.

17. Deardorff WJ and Grossberg GT: Targeting neuroinflammation in Alzheimer's disease: Evidence for NASIDs and novel therapeutics. Expert Rev Neurother 17: 17-32, 2017.

18. Miguel-Álvarez M, Santos-Lozano A, Sanchis-Gomar F, Fiuza-Luces C, Pareja-Galeano H, Garatachea N and Lucia A: Non-steroidal anti-inflammmatory drugs as a treatment for Alzheimer's disease: A systematic review and meta-analysis of treatment effect. Drugs Aging 32: 139-147, 2015. 
19. Mueller BK, Mack $\mathrm{H}$ and Teusch $\mathrm{N}$ : Rho kinase, a promising drug target for neurogical disorders. Nat Rev Drug Discov 4: 387-398, 2005

20. Petraos S, Li QX, George AJ, Hou X, Kerr ML, Unabia SE, Hatzinisiriou I, Maksel D, Aguilar MI and Small DH: The beta-amyloid protein of Alzheimer's disease increase neuronal CRMP-2 phosphorylation by a Rho-GTP mechanism. Brain 131: 90-108, 2008.

21. Bauer PO, Wong HK, Oyama F, Goswami A, Okuno M, Kino Y, Miyazaki $\mathrm{H}$ and Nukina N: Inhbition of Rho kinases enhances the degradation of mutant huntingtin. J Boiol Chem 284: 13153-13164, 2009.

22. Herskowitz JH, Feng Y, Mattheyses AL, Hales CM, Higginbotham LA, Duong DM, Montine TJ, Troncoso JC, Thambisetty M, Seyfried NT, et al: Pharmacologic inhibition of ROCK2 suppresses amyloid- $\beta$ production in an Alzheimer's disease mouse model. J Neurosci 33: 19086-19098, 2013.

23. Couch BA, DeMarco GJ, Gourley SL and Koleske AJ: Increased dendrite branching in AbetaPP/PS1 mice and elongation of dendrite arbors by fasudil administration. J Alzheimers Dis 20: 1003-1008, 2010.

24. Yu JZ, Li YH, Liu CY, Wang Q, Gu QF, Wang HQ, Zhang GX, Xiao BG and Ma CG: Multitarget therapeutic effect of fasudil in APP/PS1transgenic mice. CNS Neurol Disord Drug Targets 16: 199-209, 2017.

25. Hong S, Beja-Glasser VF, Nfonoyim BM, Frouin A, Li S, Ramakrishnan S, Merry KM, Shi Q, Rosenthal A, Barres BA, et al: Complement and microglia mediate early synapse loss in Alzheimer mouse models. Science 352: 712-716, 2016.

26. Terry RD, Masliah E, Salmon DP, Butters N, DeTeresa R, Hill R, Hansen LA and Katzman R: Physical basis of cognitive alterations in Alzheimer's disease: Synapse loss is the major correlate of cognitive impairment. Ann Neurol 30: 572-580, 1991.

27. Almeida CG, Tampellini D, Takahashi RH, Greengard P, Lin MT, Snyder EM and Gouras GK: Beta-amyloid accumulation in APP mutant neurons reduces PSD-95 and GluR1 in synapses. Neurobiol Dis 20: 187-198, 2005.

28. Huang EJ and Reichardt LF: Neurotrophins: Roles in neuronal development and function. Annu Rev Neurosci 24: 677-736, 2001.

29. Waterhouse EG and $\mathrm{Xu} \mathrm{B}$ : New insights into the role of brain-derived neurotrophic factor in synaptic plasticity. Mol Cell Neurosci 42: 81-89, 2009.

30. Prvulovic D and Hampel $\mathrm{H}$ : Amyloid $\beta(\mathrm{A} \beta)$ and phospho-tau (p-tau) as diagnostic biomarkers in Alzheimer's disease. Clin Chem Lab Med 49: 367-374, 2011.

31. Tohda C, Ichimura M, Bai Y, Tanaka K, Zhu S and Komatsu K: Inhibitory effects of Eleutherococcus senticosus extracts on amyloid beta(25-35)-induced neuritic atrophy and synaptic loss. J Pharmacol Sci 107: 329-339, 2008

32. Zhang ZH, Yu LJ, Hui XC, Wu ZZ, Yin KL, Yang $\mathrm{H}$ and $\mathrm{Xu} \mathrm{Y:}$ Hydroxy-safflor yellow $A$ attenuates $A \beta_{1-42}$-induced inflammation by modulating the JAK2/STAT3/NF- $\kappa$ B pathway. Brain Res 1563: 72-80, 2014.

33. Morroni F, Sita G, Tarozzi A, Rimondini R and Hrelia P: Early effects of A $\beta 1-42$ oligomers injection in mice: Involvement of PI3K/Akt/GSK3 and MAPK/ERK1/2 pathways. Behav Brain Res 314: 106-115, 2016.

34. Sevigny J, Chiao P, Bussière T, Weinreb PH, Williams L, Maier M, Dunstan R, Salloway S, Chen T, Ling Y, et al: The antibody aducanumab reduces $\mathrm{A} \beta$ plaques in Alzheimer's disease. Nature 537: 50-56, 2016.

35. MateoI,Vázquez-HigueraJL,Sánchez-JuanP,Rodríguez-RodríguezE, Infante J, García-Gorostiaga I, Berciano J and Combarros O: Epistasis between tau phosphorylation regulating genes (CDK5R1 and GSK-3beta) and Alzheimer's disease risk. Acta Neurol Scand 120: 130-133, 2009.

36. Goedert M: Neurofibrillary pathology of Alzheimer's disease and other tauopathies. Prog Brain Res 117: 287-306, 1998.

37. Cummings J, Aisen PS, DuBois B, Frölich L, Jack CR Jr, Jones RW, Morris JC, Raskin J, Dowsett SA and Scheltens P. Drug development in Alzheimer's disease: The path to 2025. Alzheimers Res Ther 8: 39, 2016.

38. Xin YL, Yu JZ, Yang XW, Liu CY, Li YH, Feng L, Chai Z, Yang WF, Wang Q, Jiang WJ, et al: FSD-C10: A more promising novel ROCK inhibitor than Fasudil for treatment of CNS autoimmunity. Biosci Rep 35: pii: e00247, 2015.

39. Salminen A, Suuronen T and Kaarniranta K: ROCK, PAK, and Toll of synapses in Alzheimer's disease. Biochem Biophys Res Commun 371: 587-590, 2008.
40. Bobo-Jiménez V, Delgado-Esteban M, Angibaud J, Sánchez-Morán I, de la Fuente A, Yajeya J, Nägerl UV, Castillo J, Bolaños JP and Almeida A: APC/C Cdh1-Rock2 pathway controls dendritic integrity and memory. Proc Natl Acad Sci USA 114: 4513-4518, 2017.

41. Henderson BW, Gentry EG, Rush T, Troncoso JC, Thambisetty M, Montine TJ and Herskowitz JH: Rho-associated protein kinase 1 (ROCK1) is increased in Alzheimer's disease and ROCK1 depletion reduces amyloid- $\beta$ levels in brain. J Neurochem 138: 525-531, 2016.

42. Arimura N, Ménager C, Kawano Y, Yoshimura T, Kawabata S, Hattori A, Fukata Y, Amano M, Goshima Y, Inagaki M, et al: Phosphorylation by Rho kinase regulates CRMP-2 activity in growth cones. Mol Cell Biol 25: 9973-9984, 2005.

43. Swanger SA, Mattheyses AL, Gentry EG and Herskowitz JH: ROCK 1 and ROCK2 inhibition alters dendritic spine morphology in hippocampal neurons. Cell Logist 5: e1133266, 2016.

44. Song Y, Chen X, Wang LY, Gao W and Zhu MJ: Rho kinase inhibitor fasudil protects against $\beta$-amyloid-induced hippocampal neurodegeneration in rats. CNS Neurosci Ther 19: 603-610, 2013.

45. Li YH, Yu JZ, Liu CY, Zhang H, Zhang HF, Yang WF, Li JL, Feng QJ, Feng L, Zhang GX, et al: Intranasal delivery of FSD-C10, a novel Rho kinase inhibitor, exhibits therapeutic potential in experimental autoimmune encephalomyelitis. Immunology 143: 219-229, 2014.

46. Revett TJ, Baker GB, Jhamandas J and Kar S: Glutamate system, amyloid $B$ peptides and tau protein: Functional interrelationships and relevance to Alzheimer disease pathology. J Psychiatry Neurosci 38: 6-23, 2013

47. Masoudian N, Riazi GH, Afrasiabi A, Modaresi SM, Dadras A, Rafiei S, Yazdankhah M, Lyaghi A, Jarah M, Ahmadian S and Seidkhani H: Variations of glutamate concentration within synaptic cleft in the presence of electromagnetic fields: An artificial neural networks study. Neurochem Res 40: 629-642, 2015.

48. Anggono V, Tsai LH and Götz J: Glutamate receptors in Alzheimer's disease: Mechanisms and therapies. Neural Plast 2016: 8256196, 2016.

49. Dong Z, Han H, Li H, Bai Y, Wang W, Tu M, Peng Y, Zhou L, $\mathrm{He} \mathrm{W}, \mathrm{Wu} \mathrm{X}$, et al: Long-term potentiation decay and memory loss are mediated by AMPAR endocytosis. J Clin Invest 125: 234-247, 2015

50. Hsieh H, Boehm J, Sato C, Iwatsubo T, Tomita T, Sisodia S and Malinow R: AMPAR removal underlies Abeta-induced synaptic depression and dendritic spine loss. Neuron 52: 831-843, 2006.

51. Keith D and El-Husseini A: Excitation control: Balancing PSD-95 function at the synapse. Front Mol Neurosci 1: 4, 2008.

52. Valtorta F, Pennuto M, Bonanomi D and Benfenati F: Synaptophysin: Leading actor or walk-on role in synaptic vesicle exocytosis? Bioessays 26: 445-453, 2004.

53. Poo MM: Neurotrophins as synaptic modulators. Nat Rev Neurosci 2: 24-32, 2001.

54. Lessmann V, Gottmann K and Malcangio M: Neurotrophin secretion: Current facts and future prospects. Prog Neurobiol 69: 341-374, 2003.

55. Bimonte HA, Nelson ME and Granholm AC: Age-related deficits as working memory load increases: Relationships with growth factors. Neurobiol Aging 24: 37-48, 2003.

56. Straten G, Eschweiler GW, Maetzler W, Laske C and Leyhe T: Glial cell-line derived neurotrophic factor (GDNF) concentrations in cerebrospinal fluid and serum of patients with early Alzheimer's disease and normal controls. J Alzheimers Dis 18: 331-337, 2009.

57. Forlenza OV, Miranda AS, Guimar I, Talib LL, Diniz BS, Gattaz WF and Teixeira AL: Decreased neurotrophic support is associated with cognitive decline in non-demented subjects. J Alzheimers Dis 46: 423-429, 2015.

58. Pertusa M, García-Matas S, Mammeri H, Adell A, Rodrigo T, Mallet J, Cristòfol R, Sarkis C and Sanfeliu C: Expression of GDNF transgene in astrocytes improves cognitive deficits in aged rats. Neurobiol Aging 29: 1366-1379, 2008.

This work is licensed under a Creative Commons Attribution-NonCommercial-NoDerivatives 4.0 International (CC BY-NC-ND 4.0) License. 